\title{
Desarrollo de una superficie de Fresnel tipo canal usando un LED de alta potencia para adaptarlo a un reactor fotoquímico
}

\section{Development of a channel type Fresnel surface using a high-power LED to adapt it to a photochemical reactor}

GONZÁLEZ-GALINDO, Edgar Alfredơ*, SORIANO-HERNÁNDEZ, Víctor Hugo, PÉREZGARCÍA, Jorge y HERNÁNDEZ-HERNÁNDEZ, J. Guadalupe

Universidad Nacional Autónoma de México, Facultad de Estudios Superiores Aragón, Centro Tecnológico Aragón.

ID $1^{\text {er }}$ Autor: Edgar Alfredo, González-Galindo / ORC ID: 0000-0003-4654-9595, Researcher ID Thomson: G-7927-2018, CVU CONACYT ID: 351785

ID $1^{\mathrm{er}}$ Coautor: Víctor Hugo, Soriano-Hernández / ORC ID: 0000-0002-8895-214X, Researcher ID Thomson: D-2475-2019, CVU CONACYT ID: 965077

ID $2^{\text {do }}$ Coautor: Jorge, Pérez-García / ORC ID: 0000-0002-6085-6375, CVU CONACYT ID: 1014606

ID $3^{\text {er }}$ Coautor: J. Guadalupe, Hernández-Hernández / ORC ID: 0000-0002-5093-9468, Researcher ID Thomson: G-79272018, CVU CONACYT ID: 98984

DOI: $10.35429 /$ JSL.2019.18.6.7.14

Recibido 23 Abril, 2019; Aceptado 30 Junio, 2019

\begin{abstract}
Resumen
Una superficie de Fresnel tipo canal fue diseñada usando el método de ajuste de interpolación polinomial. El arreglo experimental fue integrado por un complejo metálico, una fuente de iluminación LED de alta potencia, un sistema de enfriamiento y un concentrador de Fresnel. Las pruebas se realizaron en una cámara obscura, colocando el compuesto a una distancia focal efectiva de los rayos reflejados del concentrador, durante un tiempo. Al exponerse la muestra a la irradiación LED, sus cambios fueron registrados en un espectrofotómetro UV-Vis, obteniendo una gráfica de absorbancia contra longitud de onda, los cambios observados en las bandas de transición en desplazamiento e intensidad de la región azul al rojo del espectro, confirmaron la eficiencia y utilidad del concentrador tipo Fresnel en el desarrollo del reactor fotoquímico. Este modelo de reactor fotoquímico tiene una ventaja en comparación a los comerciales, debido a que usa muestras de compuestos en soluciones pequeñas, además de ser portátil, puede implementarse en laboratorios de universidades y farmacéuticos. Los resultados fueron favorables, se obtuvo un bajo consumo de energía, y una reducción de tiempo en el proceso de reacción molecular del compuesto analizado.
\end{abstract}

Concentrador de Fresnel, Reactor fotoquímico, Bajo consumo de energía

\begin{abstract}
A channel-type Fresnel surface was designed using the polynomial interpolation adjustment method. The experimental arrangement was composed of a metal complex, a high power LED lighting source, a cooling system and a Fresnel concentrator. The tests were done in a dark chamber, placing the compound at an effective focal distance from the reflected rays of the concentrator, for a time. By exposing the sample to the LED irradiation, its changes were registered in a UV-Vis spectrophotometer, obtaining an absorbance graph vs wavelength, the changes observed in the transition bands in displacement and intensity of the blue region to the red of the spectrum, confirmed the efficiency and usefulness of the Fresnel-type concentrator in the development of the photochemical reactor. This photochemical reactor has an advantage over commercial reactors, because it uses samples of compounds in small solutions, is portable, it can be implemented in university and pharmaceutical laboratories. The results were favorable, a low energy consumption was obtained, and a reduction of time in the molecular reaction process of the analyzed compound.
\end{abstract}

Fresnel concentrator, Photochemical reactor, Low energy consumption

Citación: GONZÁLEZ-GALINDO, Edgar Alfredo, SORIANO-HERNÁNDEZ, Víctor Hugo, PÉREZ-GARCÍA, Jorge y HERNÁNDEZ-HERNÁNDEZ, J. Guadalupe. Desarrollo de una superficie de Fresnel tipo canal usando un LED de alta potencia para adaptarlo a un reactor fotoquímico. Revista de Simulación y Laboratorio. 2019, 6-18: 7-14

*Correspondencia al Autor (Correo electrónico: unam_alf@ comunidad.unam.mx)

$\dagger$ Investigador contribuyendo como primer Autor 


\section{Introducción}

Hoy en día es de gran importancia implementar el tratamiento en sustancias toxicas emitidas al medio ambiente por la industria, para mitigar la contaminación en el impacto ambiental. Estos contaminantes pueden llegar a matar ciertos organismos ya que podrían absorberlos de manera natural, debido a grandes concentraciones, por esta razón se requiere realizar un proceso previo antes de liberar la sustancia toxica al medio ambiente, es por ello la implementación de procesos de oxidación fotoquímica y electroquímica como alternativa para el tratamiento de aguas contaminadas con sustancias antropogénicas difícilmente biodegradables, así como para eliminar bacterias en aguas destinadas al consumo humano.

Los resultados corroboran la eficiencia de la electroquímica y la fotocatálisis para destruir contaminantes biológicos y químicos, lo cual aumenta la calidad del agua. La combinación de estos procesos, como tratamiento preliminar, seguida por un proceso biológico, es muy prometedora desde el punto de vista económico (Sarria, Parra, Rincón, Torres, \& Pulgarín, 2005).

Los reactores fotoquímicos son empleados en el tratamiento de Percolado de relleno sanitario (estiércol) y para evaluar su potencial de reúso en la agricultura. El proceso fotoquímico está basado en la acción de radiación en el espectro visible y dentro de este rango se encuentra el ultravioleta $(U V)$ aplicado al agua oxigenada llamado peróxido de hidrogeno $\left(\mathrm{H}_{2} \mathrm{O}_{2}\right)$.

Se han hecho análisis de identificación de toxicidad aguda utilizando la especie Lactuca Sativa (lechuga) y el estiércol tratado por proceso fotoquímico $\left(\mathrm{H}_{2} \mathrm{O}_{2} / \mathrm{UV}\right)$. En las condiciones empleadas en este estudio, presenta gran potencial para ser probado en pequeñas dosis en procedimientos de corrección de la acidez del suelo (Rosolem Chinelatto, Greve, Teixeira Pelegrini, \& de Brito, 2015).

Los reactores fotoquímicos o la degradación fotoquímica se utilizan en la industria textil para la reutilización del agua obtenida de un efluente tratado por $U V$ y $\mathrm{H}_{2} \mathrm{O}_{2}$.
Los valores de $p H$ utilizados en la descomposición del colorante esta se encuentra en el rango de la longitud de onda de $\lambda$ máx $=580$ $n m$, vía fotocatálisis con el $\mathrm{H}_{2} \mathrm{O}_{2}$ en presencia de luz $U V$, ha demostrado que el $\mathrm{pH}$ no interfiere en esa banda, después del tratamiento con $U V$ y $\mathrm{H}_{2} \mathrm{O}_{2}$, los tres efluentes obtenidos fueron reutilizados en el teñido de colores oscuros, bajo los estándares de calidad exigidos por la industria (Marcos Rosa, Baptista, \& Curvelo Santana, 2010).

Las superficies de Fresnel como reflectores de iluminación se pueden implementar en los reactores fotoquímicos para dirigir o redirigir la irradiancia hacia un compuesto químico a una Distancia Focal Efectiva (DFE), por ello es de vital importancia conocer la ecuación de la superficie que se utilizará. Hoy en día se utilizan superficies de forma libre utilizando distintos métodos, los principales métodos para la construcción de curvas planas son la interpolación polinomial, el ajuste por mínimos cuadrados, las curvas Bezier, las funciones splines, las curvas racionales(Delgado Olmos, 2006) y polinomio de interpolación de Newton(Aranda, 2003).

El diseño y el ajuste del contorno desconocido uno de los método de ajuste polinomial de Lagrange (Bertsekas, 2014), este método basta con tener al menos tres o más puntos de coordenadas para generar la superficie y reconstruir una superficie de Fresnel de revolución o de canal de forma libre tipo que se utilizan como concentradores o reflectores (Gonzalez, 2018). Usar el método de interpolación de Lagrange (Montiel \& Cantoral, 2003) al reconstruir un superficie y llevarlo a un reflector de Fresnel presenta algunas ventaja como un espesor más delgado y son más ligeros y su desventaja no son simétricos en comparación con las superficies parabólicos, hiperbólicos y círculos de revolución, estos últimos son simétricos y su desventaja que son pesados. (E- Alfredo, 2018)

\section{Objetivos}

Optimizar la rapidez de reacción que tiene un reactor fotoquímico, usando diodo emisor de luz (LED) de alta potencia como fuente de iluminación, utilizando un reflector de Fresnel tipo canal, el cual concentrará los rayos reflejados de la fuente al compuesto de rutenio II $\left[\mathrm{RuLClP}\left(\mathrm{Ph}_{3}\right)\right] \mathrm{Cl}$. 


\section{Hipótesis}

En este proyecto se implementa una superficie libre de forma como reflector de Fresnel tipo canal en un reactor fotoquímico, si se concentra la energía luminosa de una fuente proveniente de un $L E D$ de alta potencia a un compuesto químico, entonces se podrá reducir el consumo de energía y el tiempo invertido en su funcionamiento, además de obtener un menor costo en el uso de los reactores fotoquímicos.

\section{Metodología}

El proyecto experimental fue desarrollado en el Laboratorio de Medición e Instrumentación y Control, del Centro Tecnológico Aragón (CTA); este proyecto permite el ahorro de energía y costo en reactores fotoquímicos, así como la reducción de tiempo de trabajo, ya que en el mercado los costos son elevados y consumen gran cantidad de potencia, debido a las fuentes de iluminación utilizadas en la irradiancia de los compuestos químicos.

Para la elaboración de la superficie plano convexa(Ferri, 2012) que se observa en la Figura 1, se utilizó los siguientes instrumentos: 1 . Un flexómetro para medir un marco con dimensiones de $280 \mathrm{~mm}$ de ancho y $400 \mathrm{~mm}$ de largo, usando una superficie plana con una perforación de $161 \mathrm{~mm}$ de diámetro, 2. Se cubrió de un polímero plástico elástico, 3. Se Colocando agua $\left(\mathrm{H}_{2} \mathrm{O}\right)$ con una pipeta graduada sobre la superficie y, por acción de la gravedad, se formó la superficie plano-cóncava traslucida, 4.

Con una fuente de iluminación se proyectó los rayos incidentes, haciéndolos pasar atreves de la superficie y generando los rayos refractados para obtener la $D F E$, empleando una pantalla como plano paraxial que es perpendicular al eje óptico.

Las proyecciones de los rayos incidentes que interseca a la superficie cóncava se toman en cuenta, puesto que la normal a la superficie será perpendicular a la tangente, proyectando el rayo refractado que interseca al eje óptico y se extiende al plano paraxial.

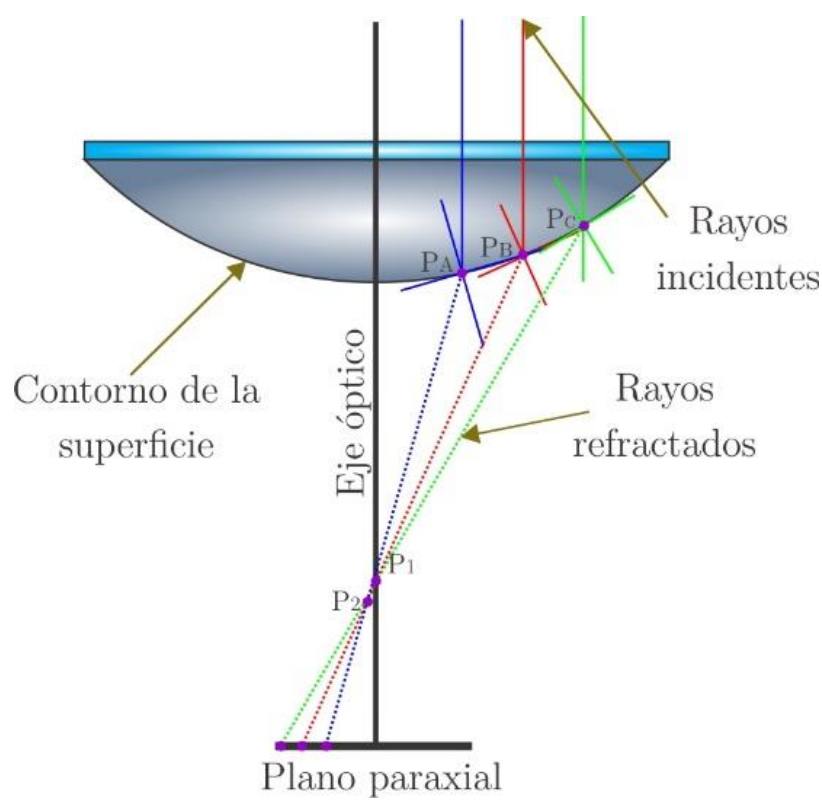

Figura 1 Diseño de la superficie plano-convexa generado por acción de la gravedad provocado por el peso del agua $\left(\mathrm{H}_{2} \mathrm{O}\right)$

Para obtener el contorno de la superficie se utiliza el método de interpolación polinomial, este método en general está basado en procedimientos matriciales, partiendo de la expresión general para la función polinomial de grado $n$, es:

$$
(f x)=a_{0}+a_{1} x+a_{2} x^{2}+\ldots+a_{n} x^{n}
$$

Este método indica que son arreglos matriciales y están definidos por la Ecuación 2 teniendo la expresión de igualdad $(A)(a)=y$ cuando despejamos al coeficiente $a$ la relación expresada es un producto de la matriz transpuesta y la nueva expresión es $(a)=y\left(\frac{1}{A}\right)$

$\mathrm{A}=\left[\begin{array}{ccccc}1 & \mathrm{x}_{0} & \mathrm{x}_{0}^{2} & \ldots & \mathrm{x}_{0}^{\mathrm{n}} \\ 1 & \mathrm{x}_{1} & \mathrm{x}_{1}^{2} & \ldots & \mathrm{x}_{1}^{\mathrm{n}} \\ \vdots & \vdots & \vdots & \ldots & \vdots \\ 1 & \mathrm{x}_{\mathrm{n}} & \mathrm{x}_{\mathrm{n}}^{2} & \ldots & \mathrm{x}_{\mathrm{n}}^{\mathrm{n}}\end{array}\right] ; a=\left[\begin{array}{c}a_{0} \\ a_{1} \\ \vdots \\ a_{\mathrm{n}}\end{array}\right] ; \mathrm{y}=\left[\begin{array}{c}\mathrm{y}_{0} \\ \mathrm{y}_{1} \\ \vdots \\ \mathrm{y}_{\mathrm{n}}\end{array}\right]$

En este caso particular el procedimiento utiliza cinco puntos de coordenadas para obtener los coeficientes del polinomio, es importante mencionar que, para obtener el grado polinomial de forma intuitiva, se podrá usar la siguiente expresión; $n=(p-1)$, donde $p$ son los puntos coordenadas y $n$ es el grado del polinomio. Para este caso se utilizó cinco puntos de coordenadas, obteniendo un polinomio de cuarto orden como se muestra en la Ecuación 3.

$f(x)=53.27 \times 10^{-6} x^{4}+66.64 \times 10^{-6} x^{3}-$ 


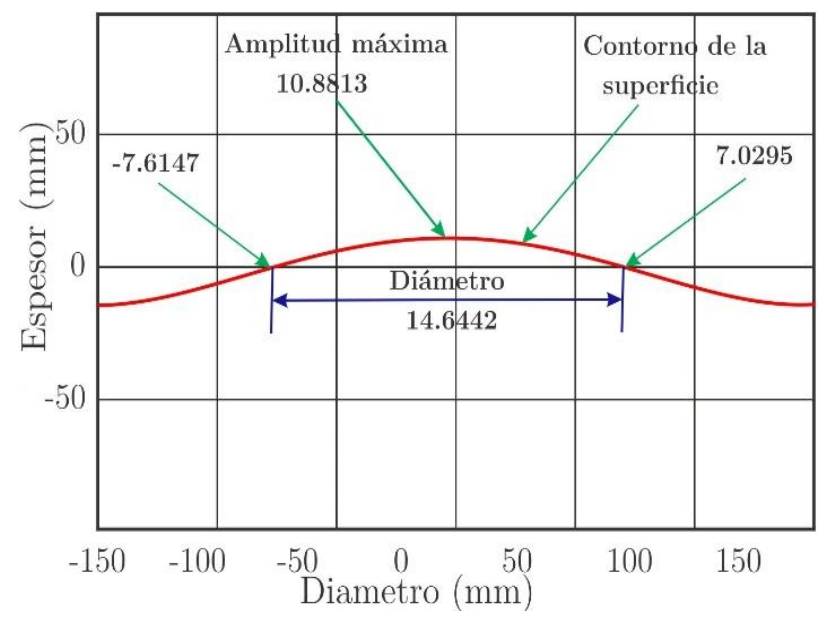

Gráfica 1 Contorno de la superficie obtenida por el método de interpolación polinomial de cuarto orden

La superficie de la Gráfica 1 se secciono en nueve fragmentos equidistantes y cada uno se desplazo al origen sobre el eje $x$, formando la superficie de Fresnel como se muestra en la Gráfica $2 \mathrm{El}$ valor de los ángulos formados entre el rayo incidente, refractado, la tangente y la normal son los siguientes: el ángulo entre el rayo incidente y la tangente es de $60^{\circ}$, el rayo incidente y la normal es de $149^{\circ}$, el rayo refractado y el eje óptico es de $150^{\circ}$, la normal y el eje óptico es de $32^{\circ}$ la tangente y el rayo refractado es de $31^{\circ}$.

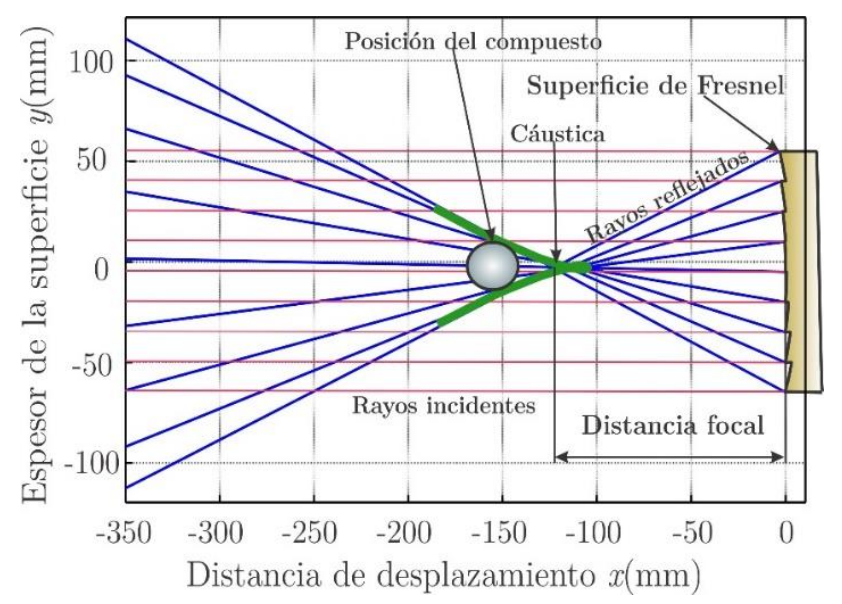

Grafica 2 Proyección de los rayos incidentes y reflejados en la superficie de Fresnel, colocando la muestra del compuesto fuera de la distancia focal

En la Grafica 2 se observa, los rayos incidentes que al reflejarse con la superficie de Fresnel tipo canal, en la acumulación de rayos se forma una envolvente llamada cáustica(Fetter, 2015) que se encuentra a partir del punto focal, se observa que el compuesto químico está colocado fuera de la distancia focal efectiva, que es donde se concentrará la mayor cantidad de energía luminosa, con el fin de exitarlo y reducir el tiempo de exposición se colocará en el círculo de máxima confusión pasando el punto focal.
Marzo 2019 Vol.6 No.18 7-14

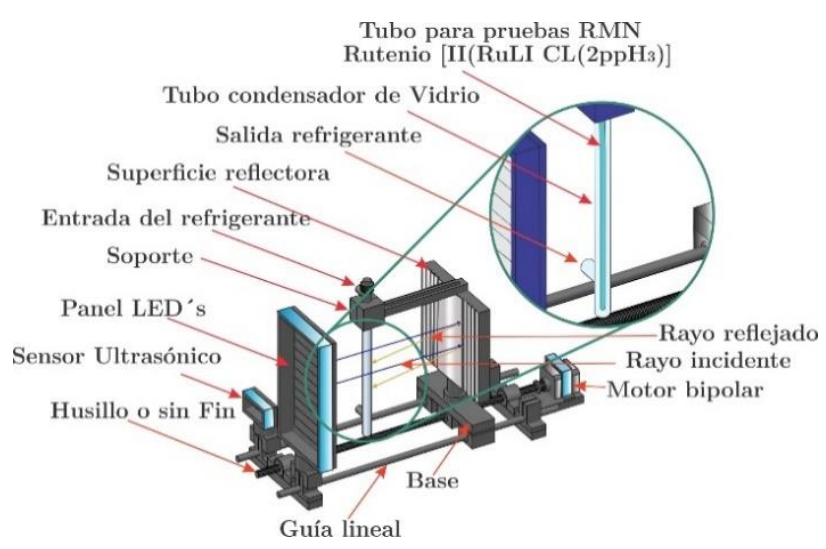

Figura 2 Diseño del reactor fotoquímico con desplazamiento de la superficie y el compuesto químico con un panel LED como fuente de iluminación

En la Figura 2 se muestra el diseño del reactor fotoquímico con la implementación del panel $L E D$ y el reflector de Fresnel tipo canal, en una base soportada por dos guías lineales y un tornillo sin fin adaptado a un motor bipolar a pasos, que desplazara a la superficie reflectora junto con la adaptación de otra base como soporte, diseñado en un programa asistido por computadora impreso y modelado en $3 D$, que soporta el tubo para pruebas en RMN. Se acercará o alejará el reflector de la fuente de iluminación para captar la gran cantidad de rayos reflejados al complejo de rutenio $l l$ $\left[R u L C l\left(P P h_{3}\right)\right]$, este tubo se encuentra dentro de un condensador de vidrio, donde fluye agua que actúa como refrigerante para regular la temperatura. Cada una de las medidas se obtuvieron utilizando un vernier para realizar las medidas y obtener las distancias en arreglo experiemntal.

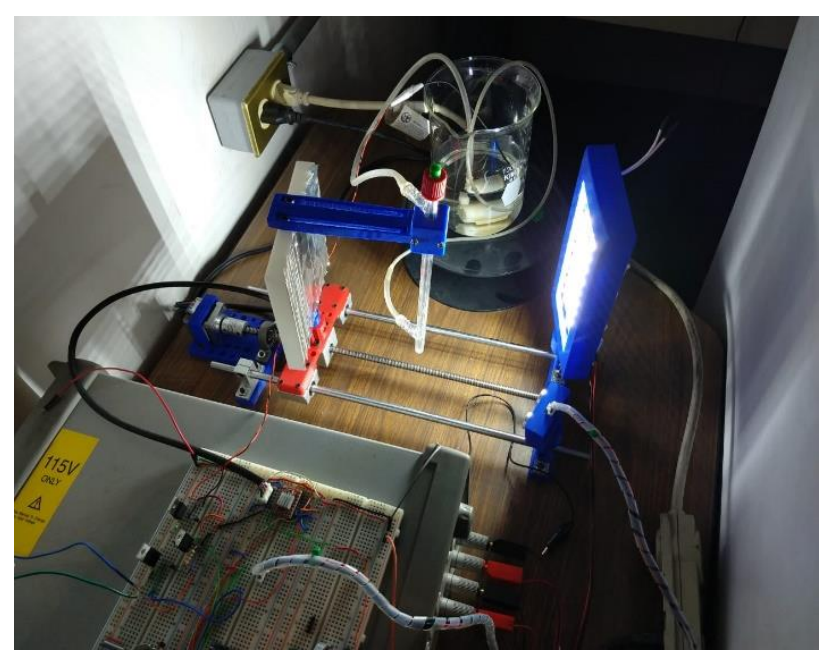

Figura 3 Reactor fotoquímico arreglo experimental adaptando una superficie de Fresnel y un panel LED como fuente de iluminación 
El diseño es un diagrama explicativo del sistema implementado en el arreglo experimental mostrado en la Figura 3, utilizando un panel de $L E D$ con una conexión serieparalelo del arreglo de treinta $L E D$ 's alimentado con $12 \mathrm{~V}$, con una corriente $I=0.516 \mathrm{~A}$ en su conexión paralelo y con un consumo de potencia de $P=6.192$ Watts, la conexión del arreglo serie con tres diodos emisores de luz consume una corriente $I=0.061 \mathrm{~A}$ con un consumo de potencia $P=0.732$ Watts y cada diodo emisor de luz consume una potencia de $P=0.24$ Watts con una corriente de $I=0.020 \mathrm{~A}$.

Se muestra en el diseño una modificación para la Figura 4, se sustituye el panel de $L E D$ por un LED de alta potencia de 30 Watts, como se muestra en la Figura 5, utilizando el mismo arreglo experimental para la irradiancia del complejo de rutenio II [RuLCl(PPh $)$ ], usando el mismo procedimiento se desarrollaron las mismas pruebas para la captura de datos y su análisis grafico con el mismo compuesto

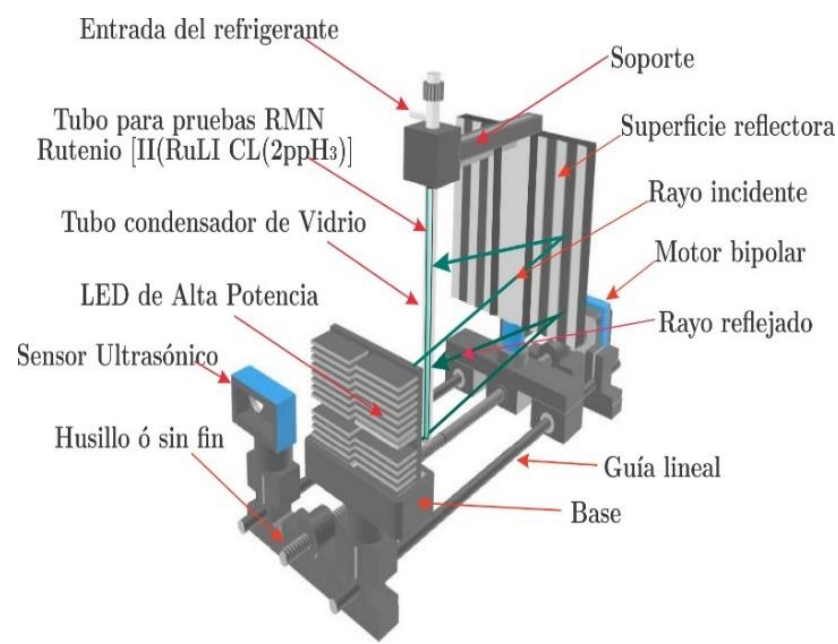

Figura 4 Diseño del reactor fotoquímico con desplazamiento de la superficie y el compuesto químico con un LED de alta potencia como fuente de iluminación

En la parte de refrigeración se utilizó una bomba sumergible y un vaso de presipitado como contenedor del refrigerante en el arreglo experimental, como se muestra en la Figura 3 y Figura 5, usando la misma tarjeta de la Figura 6, se suministró un voltaje de $12 \mathrm{~V}$ para alimentar al circuito integrado TL494CN que genera una modulación por ancho de pulso (PWM) para controlar el caudal del refrigerante. La alimentación del voltaje para el control del caudal es de $3.8 \mathrm{~V}$ con un flujo de corriente de 8 $m A$ y una potencia de $30.4 m W$, para una estabilidad en la temperatura y poder reducir el tiempo de trabajo.

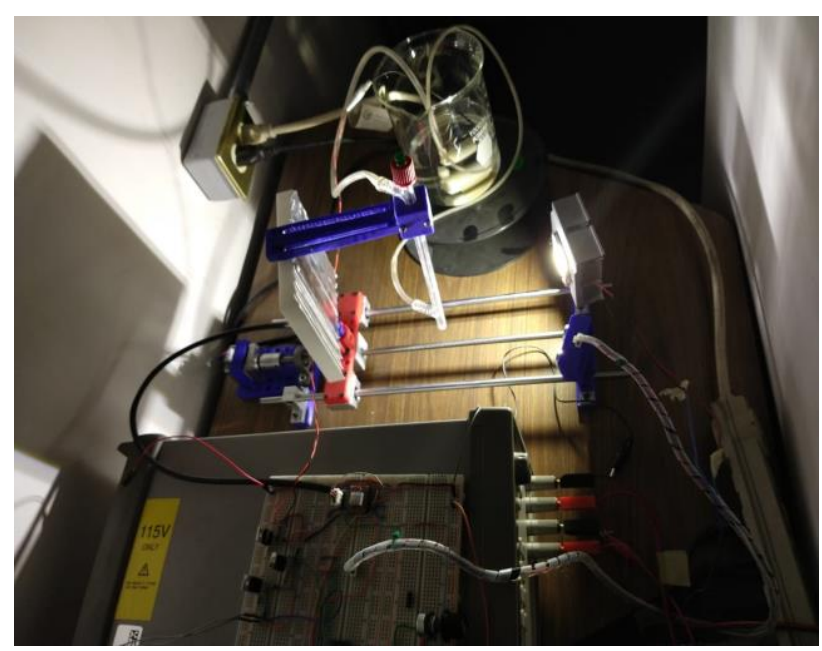

Figura 5 Reactor fotoquímico arreglo experimental adaptando una superficie de Fresnel y un LED de alta potencia como fuente de iluminación

En la Figura 6 se observa el diseño de la tarjeta impresa para el control del sistema de iluminación y el sistema de refrigeración, presenta una bornera para la alimentación del encapsulado $T L 494 C N$, en la etapa de potencia presenta una bornera para suministrar un voltaje de $0 \mathrm{~V}$ hasta $50 \mathrm{~V}$, se colocó un zócalo de 16 pines para montar el circuito integrado, se utilizaron dos MOSFET IR3205 para dos etapas de potencia con salidas independientes, al otro extremo presenta dos borneras de alimentación por si se desea suministrar la conexión a otros módulos de $12 \mathrm{~V}$ y $50 \mathrm{~V}$, en el mismo extremo se tiene las dos borneras de conexión a los diodos emisores de luz $(L E D \prime s)$ de alta potencia, se colocaron dos diodos de protección para la etapa de potencia en cada salida y un optoacoplador para proteger el TL494CN, se colocó un potenciómetro de $10 k$ para controlar la intensidad lumínica del $L E D$ y dos borneras para observar en un osciloscopio la modulación por ancho de pulso.

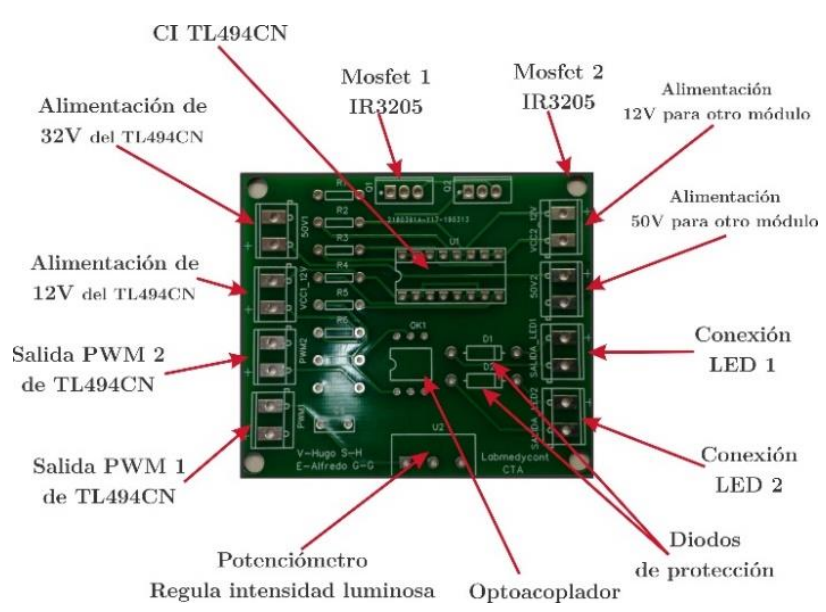

Figura 6 Diseño de una tarjeta impresa para controlar la fuente de iluminación y temperatura 
Se realizaron conexiones de la tarjeta $P C B$ con los elementos electrónicos montados, usando dos fuentes de alimentación, una de ellas se utilizó para alimentar el circuito integrado con $12 \mathrm{~V}$ (Rosolem Chinelatto et al.) $T L 494 C N$ y la segunda para suministrar con un voltaje de $30 \mathrm{~V}$ para la etapa de potencia que requiere el $L E D$ de alta potencia y colocando un disipador con un ventilador que permite un equilibrio térmico, prolongando la vida útil de la fuente de iluminación, como se observa en la Figura 7.

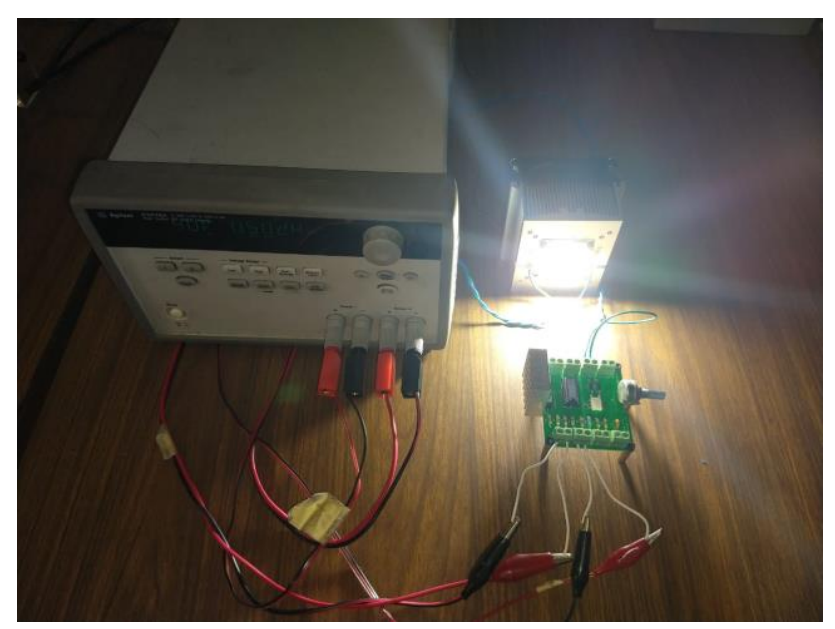

Figura 7 Diseño de una tarjeta impresa para controlar la fuente de iluminación y la bomba para controlar la temperatura

El diseño del circuito esquemático que se utilizó para el control del ciclo de trabajo del modulador por ancho de pulso PWM, que permite el control de la irradiancia de un reactor fotoquímico y el control del flujo del refrigerante del condensador de vidrio, se muestra en la Figura 8, bajo el requerimiento del diseño del reactor fotoquímico se observa en la Figura 2, además presenta las borneras de alimentación y de salida de control, colocado un optoacoplador para la protección en la etapa de potencia.

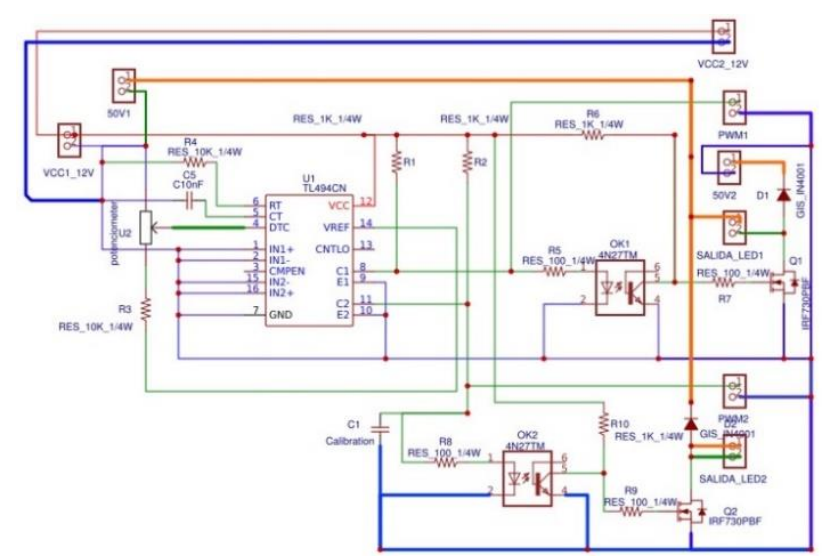

Figura 8 Circuito esquemático para el control del sistema de iluminación
Cuando se trabaja con diodos emisores de luz, se debe de tomar en cuenta su ángulo de apertura, ya que estos pueden variar dependiendo del fabricante.

\section{Resultados}

En la Gráfica 3 se observa el comportamiento del complejo de rutenio II [RuLCl(Ph3)] Cl, después de ser irradiado durante 30, 60, 90 y 120 minutos por un panel LED de 4.4 Watts de potencia sin usar el reflector de Fresnel tipo canal a una distancia de $200 \mathrm{~mm}$.

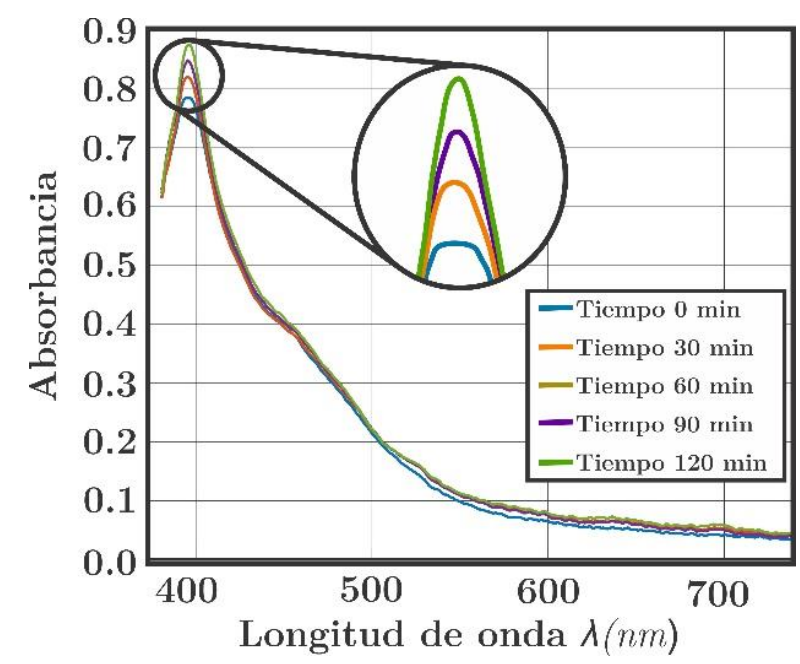

Gráfica 3 Comportamiento del espectrofotómetro Vis sin el uso del reflector de Fresnel tipo canal con una fuente de 4 Watts

En la Gráfica 4 se observa el comportamiento del complejo de rutenio II [RuLCl(Ph 3)] Cl, colocando un reflector de Fresnel tipo canal después de ser irradiado con la misma fuente de iluminación durante 120 minutos, por un panel LED de 4.4 Watts de potencia, se puede apreciar que la amplitud se incrementa y se desplaza conforme al tiempo la longitud de onda.

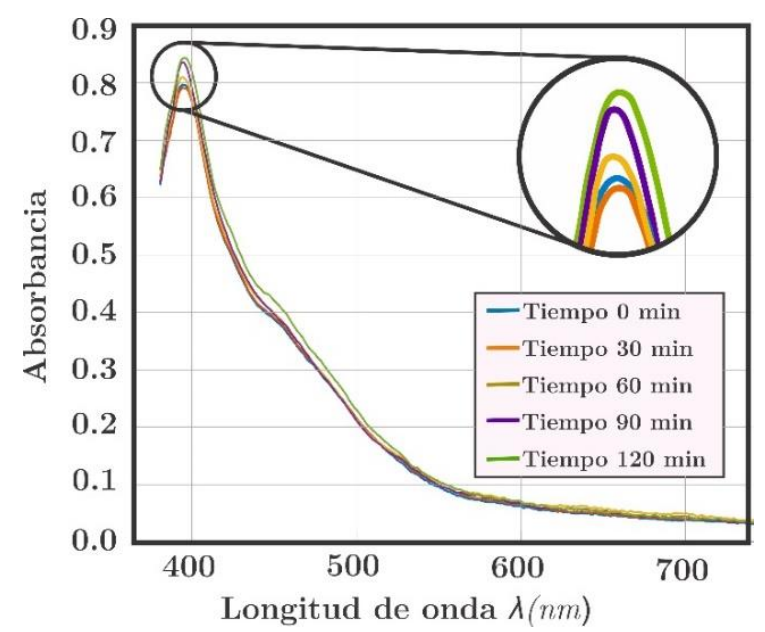

Gráfica 4 Comportamiento del espectrofotómetro Vis usando el reflector de Fresnel tipo canal con una fuente de 4 Watts

GONZÁLEZ-GALINDO, Edgar Alfredo, SORIANO-HERNÁNDEZ, Víctor Hugo, PÉREZ-GARCÍA, Jorge y HERNÁNDEZ-HERNÁNDEZ J. Guadalupe. Desarrollo de una superficie de Fresnel tipo canal usando un LED de alta potencia para adaptarlo a un reactor fotoquímico. Revista de Simulación y Laboratorio. 2019 
La Gráfica 5, muestra el uso de una fuente de iluminación con un $L E D$ de alta potencia de 30 Watts, causa un comportamiento en el complejo de rutenio II [RuLCl(Ph3)] Cl ya que hay una diferencia notable en la amplitud y en el desplazamiento en la longitud de onda después de ser irradiado durante 120 minutos y al ser analizado en el espectrofotómetro Vis, en este caso al reactor fotoquímico se adaptó el reflector de Fresnel tipo canal.

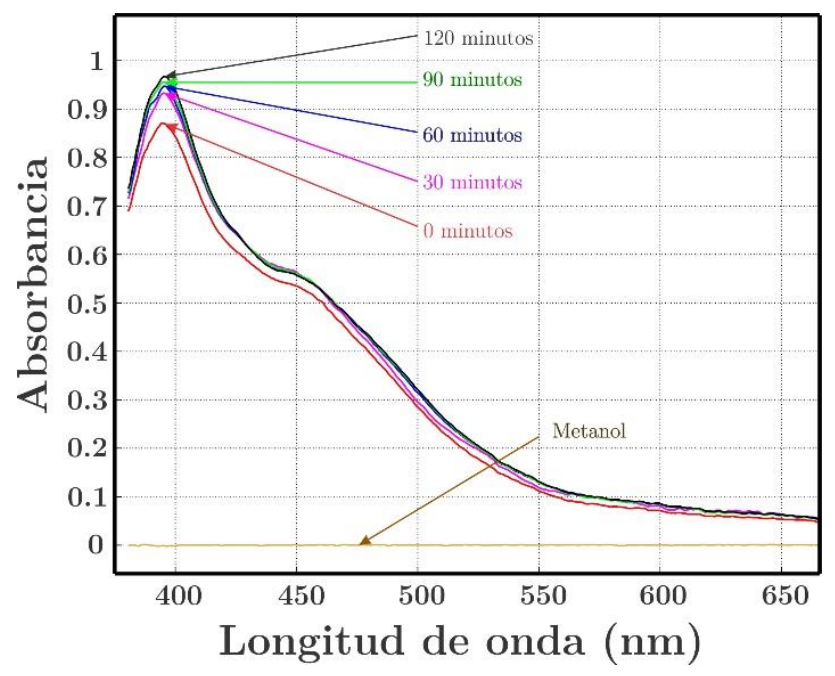

Gráfica 5 Comportamiento del espectrofotómetro Vis usando un reflector de Fresnel tipo canal con una fuente de 33 Watts

La amplitud nos indica una reacción en el compuesto químico, dependiendo de la variación sobre el eje de la absorbancia y si hay un desplazamiento sobre el eje de la longitud de onda hacia el rango del espectro visible nos indica si el compuesto es fotoquímico.

\section{Conclusión}

Con base en el estudio y las pruebas realizadas, se observó que adaptar un reflector de Fresnel tipo canal fue más favorable, ya que al irradiar el compuesto químico, haciendo uso de dicha superficie libre de forma, concentrando los rayos incidentes que provienen de la fuente de iluminación del $L E D$ de alta potencia, actúa como una segunda fuente que capta los rayos incidentes y los refleja concentrándolos al compuesto de rutenio II [RuLClP(Ph 3)] Cl, y se observo en la gráfica un desplazamiento en el eje de la longitud de onda y una amplitud mayor sobre el eje de absorvancia, que cuando no se utilizó el reflector.
De esta manera se comprobó que, al introducir una superficie como concentrador de energía luminosa en los reactores fotoquímicos, reduce el consumo de energía utilizada para su funcionamiento, además de disminuir el tiempo de trabajo para llegar a un punto establecido de alteración molecular del compuesto químico, y de esta manera poder reducir el consumo de energía de los reactores. Además, se afirma que este tipo de innovación aporta un avance significativo a los análisis químicos, esta tecnología actual potencializa y mejora el funcionamiento de los reactores además de ayudar al medio ambiente.

\section{Agradecimiento}

Los autores agradecen al Centro Tecnológico de la Facultad de Estudios Superiores Aragón de la Universidad Nacional Autónoma de México, por las facilidades en el uso del Laboratorio de Medición e Instrumentación y Control, al Laboratorio de Ingeniería Ambiental y al Programa de Apoyo a Proyectos para la Innovación y Mejoramiento de la Enseñanza (PAPIME) con No. de clave PE105519, también se agradece a Omar-Y Espinosa-L, Oscar-A Luy-M, Laura Vázquez-Z y Douglas-K Soto-D por su colaboración técnica.

\section{Referencias}

Aranda, D. F. C. (2003). Introducción a los Métodos Numéricos: Software en Basic y aplicaciones en hidrología superficial: UASLP. Bertsekas, D. P. (2014). Constrained optimization and Lagrange multiplier methods: Academic press.

Delgado Olmos, Á. H. (2006). Modelado de superficies polinomiales y su aplicación a la técnica: Universidad de Granada.

E- Alfredo, G.-G. (2018). Diseño de una superficie de revolución libre de forma impreso en 3D como concentrador solar tipo Fresnel. Congreso Internacional de Investigación e Innovación 2018, 3, 8377-8388.

Ferri, J. (2012). Análisis del Comportamiento Aerodinámico de Perfiles. In: Valencia: Universitat Politecnica de Valencia.

Fetter, H. L. J. X. C. V. N.-L. (2015). Curvas que se pintan solas. 105 . 
Gonzalez, E. A. (2018). Diseño de una superficie cuadrada como concentrador solar de revolución de forma libre tipo Fresnel impreso en 3D Design of a square surface as a solar concentrator of Fresnel type revolution printed in 3D. Revista del Desarrollo Tecnológico, 2, 8.

Marcos Rosa, J., Baptista, E. A., \& Curvelo Santana, J. C. (2010). Degradação fotoquímica e reuso da água obtida de um efluente têxtil tratado via UV/H2O2. Exacta, 8(2), 169-178.

Montiel, G., \& Cantoral, R. J. N. R. d. D. d. 1. M. (2003). Una presentación visual del polinomio de Lagrange. 55, 3-22.

Rosolem Chinelatto, M., Greve, L. F., Teixeira Pelegrini, R., \& de Brito, N. N. (2015). Tratamento Fotoquímico de Percolado de Aterro Sanitário Visando Reuso na Agricultura. Eclética Química, 40, 141-154.

Sarria, V. M., Parra, S., Rincón, Á. G., Torres, R. A., \& Pulgarín, C. (2005). NUEVOS SISTEMAS ELECTROQUÍMICOS Y FOTOQUÍMICOS PARA EL TRATAMIENTO DE AGUAS RESIDUALES Y DE BEBIDA. Revista Colombiana de Química, 34(2), 161173. 